\title{
Poly (2-Hydroxyethyl Methacrylate) Macroporous Cryogel for Extracorporeal Medical Devices
}

\author{
Wuraola Akande ${ }^{1,2, ~ *, ~ L y u b a ~ M i k h a l o v s k a ~}{ }^{1}$, Stuart James $^{1}$, Sergey Mikhalovsky ${ }^{1,3}$ \\ ${ }^{1}$ Biomaterials and Medical Devices Research Group, School of Pharmacy and Biomolecular Sciences, University of Brighton, Brighton, UK \\ ${ }^{2}$ Department of Clinical Pharmacy and Pharmacy Administration, Faculty of Pharmacy, University of Ibadan, Ibadan, Nigeria \\ ${ }^{3}$ School of Engineering, Nazarbayev University, Astana, Kazakhstan
}

Email address:

wa20@brighton.ac.uk (W. Akande),wuradol@gmail.com (W. Akande), L.Mikhalovska@brighton.ac.uk (L. Mikhalovska), S.L.James@brighton.ac.uk (S. James), s.mikhalovsky@brighton.ac.uk (S. Mikhalovsky), smikhalovsky@nu.edu.kz (S. Mikhalovsky)

\section{To cite this article:}

Wuraola Akande, Lyuba Mikhalovska, Stuart James, Sergey Mikhalovsky. Poly (2-Hydroxyethyl Methacrylate) Macroporous Cryogel for Extracorporeal Medical Devices. International Journal of Biomedical Materials Research. Vol. 3, No. 4, 2015, pp. 46-55. doi: 10.11648/j.ijbmr.20150304.12

\begin{abstract}
Poly (2-hydroxyethyl methacrylate) PHEMA monolithic cryogels were synthesized by free radical polymerization at $-12^{\circ} \mathrm{C}$ for 18 hours and produced spongy, elastic and macroporous gel matrix. Scanning Electron Microscopy (SEM) measured structural properties of PHEMA monolithic cryogel matrix to visualize pore morphology. Mechanical properties of PHEMA monolithic cryogel such as storage modulus, compressive modulus, and creep test were measured with Dynamic mechanical analyzer (DMA). The PHEMA monolithic cryogel matrix shows $\sim 97 \%$ recovery after $70 \%$ compression of cryogel and has a compressive modulus of $1.8 \mathrm{kPa}$ to $8.5 \mathrm{kPa}$.
\end{abstract}

Keywords: Macroporous Cryogel, Poly (2-Hydroxyethyl Methacrylate), Mechanical Properties, Creep Test, Compressive Modulus

\section{Introduction}

Cryogels are macroporous polymeric materials that can be synthesized by free radical polymerization. Briefly, free radical polymerization is controlled by the composition of initiating system in the reaction mixture. Concentrations of the initiator ammonium persulphate (APS) and activator N, $\mathrm{N}, \mathrm{N}, \mathrm{N}$-tetramethylethylenediamine (TEMED) has a major impact on the polymerization rate as well as on molecular weight of the resulting polymers. These processes take place at sub-zero temperatures [1]. The solvent (water) is frozen while the dissolved chemicals (monomers, cross-linkers and initiators/activator system) are concentrated in small nonfrozen regions, referred to as 'non-frozen liquid micro phase' where polymerization occurs. Formation of the gel occurs in the liquid phase and the ice crystals of frozen solvent serve as pore agent. After melting the ice crystals, the shape and size of the ice crystals determine the shape and size of the pores formed, hence a system of large interconnected pores is formed [2]. Macroporous monolithic cryogels with large interconnected pore size range between $10 \mu \mathrm{m}$ and $200 \mu \mathrm{m}$ are heterophase systems where solvent (water) is present both inside interconnected pores and bound to the polymer network [3-5].

Poly (2-hydroxyethyl methacrylate) (PHEMA) is a well studied polymer widely used for many biomedical applications, ranging from production of contact lenses [6] and wound dressing [7] to controlled release and drug delivery devices [8] as well as for the development of blood compatible surfaces [9].

Presently, there is a rising interest in macroporous polymer cryogels due to their distinctive various open porous structures, which considerably increases their equilibrium sorption properties and allows unrestricted diffusion of solutes, nano - and even microparticles [10-12]. Macroporous cryogels have previously been used for biomedical applications and protein purification [3, 13-17]. Cryogels have a unique combination of properties such as interconnected pores of $10-200 \mu \mathrm{m}$ size, porosity exceeding 90\%, high mechanical and chemical stability, elasticity and sponginess, drying and rehydrating without impairing pore structures $[1,18]$.

Mechanical properties of polymeric materials which are the strength of today's drug delivery and biomedical devices [19] is of particular importance as these have been shown to 
directly affect clinical efficacy [20]. Therefore the successful development of such products requires extensive characterization and also comprehensive understanding of the polymeric material. These properties may be understood using a range of techniques including tensile analysis, thermomechanical analysis, thermodilatometry and the dynamic mechanical analyzer (DMA). These techniques used to measure the mechanical properties of polymeric materials involve application of an external force whilst measuring the resulting deformation of the material.

DMA is the most easily accessible, providing a rapid and non-destructive way of quantifying the rheological/mechanical properties of polymers. The application of DMA for drug delivery and biomedical devices characterization has gained increased attention over the last decade. Comprehensive mathematical theories of the dynamic mechanical analyzer have been previously described [21-23]. The dynamic mechanical analyzer is an analytical technique in which oscillatory stress (the force per unit area (in Pascals, $\mathrm{Pa}$ ) required to deform the sample) is applied to the sample and the resultant strain (the amount by which the sample is deformed) selected from within the linear viscoelastic region and the result is determined as a function of both temperature and frequency [23]. Dynamic mechanical methods characterize the viscoelastic properties (simultaneous existence of viscous 'fluid' and elastic 'solid' properties in all materials) [24], such as storage and loss modulus (the resistance of a material to deformation, in $\mathrm{Pa}$ ) [21]. Viscoelastic systems after application of a constant stress, generally do not maintain constant deformation, but continue to deform as a function of time. [21, 22, 25]. This occurrence is referred to as creep. In flow process, viscoelastic liquid could store some of the applied energy and use this energy to partially recover from the stressinduced deformation. Hooke's law states that the ratio of stress $(\sigma)$ to strain $(\gamma)$ in an elastic material is constant, within a specific elastic limit. The constant of proportionality is defined as Young's modulus (E), which is calculated by equation

$$
\frac{\text { Stress }}{\text { Strain }}=\frac{\sigma}{\gamma}
$$

Equation 1

The behavior of elastic solids may be represented using an extendable spring whereby the load applied to the spring represents the stress and the extension represents the strain. The load applied to the spring is directly proportional to the extension, which in the case of elastic solid is instantaneous; and time independent. On the removal of the load, the stored elastic energy is used to return the spring to its original position; this response is also instantaneous [26]. Deviations from ideal elastic behaviour occur whenever the elastic limit is exceeded [24]. 2-hydroxyethyl methacrylate (HEMA) was chosen in this study as the basic component because of its chemical and biological stability and biocompatibility [27].

The dynamic mechanical analyzer determined mechanical properties of epoxy monolithic PHEMA cryogel. The objective of this study is to help improve understanding on the mechanical properties of poly (2-hydoxylethyl methacrylate) macroporous cryogels. The dynamic mechanical analysis for compressive modulus, creep test and cyclic compression analyzer has been demonstrated for the first time to be useful in the evaluation of the elastic and spongy-like properties of macroporous polymeric cryogels. Thus, the data produced in this report can provide a technological platform for development and/or use of HEMA macroporous monolithic cryogel as a potential matrix for cell separation and bio-separation in an extracorporeal apheresis system.

\section{Materials and Methods}

2- hydroxyethyl methacrylate, (HEMA 98\%, stabilized), allyl glycidyl ether (AGE 99\%), and N, N-methylene bisacrylamide, (MBAA 96\%) were purchased from Acros Organics UK. N, N, N', N'-tetramethyethylenediamine electrophoresis grade (TEMED 97\% Fisher Bio reagents) and ammonium persulfate (APS) were purchased from Fisher Scientific UK.

Dimethyl sulfoxide K 40270531933 and Fluorescein 5(6)-isothio-cyanate, mixed isomers (FITC 90\%) from Sigma, conical flasks, vacuum pump, eppendorf tubes and glass columns were used. Confocal laser scanning microscope (Zeiss LSM410), cryobath (Julabo Ltd UK), scanning electron microscope (JOEL JSM -5600LV), Dynamic mechanical analyzer DMA 2980 by TA Instruments, Inc. (USA), Sample clamp: Compression plates $(15 \mathrm{~mm}$ diameter) and Image J software were used.

\subsection{Cryogel Synthesis}

An epoxy containing PHEMA cryogel was produced from monomers of 2-hydroxyethyl methacrylate (HEMA $5.28 \mathrm{ml}$ ) and allyl glycidyl ether (AGE $1.08 \mathrm{ml}$ ), N, N'methylenebisacrylamide (MBAAm $1.342 \mathrm{~g}$ ) was used as a cross linker. These were dissolved in deionized water $92.2 \mathrm{ml}$ (final concentration of monomers $8 \% \mathrm{w} / \mathrm{v}$ ), monomers ratio to MBAAm 6:1. The resulting solutions were degassed for about 20 minutes by using $\mathrm{N}_{2}$ gas or water pipe vacuum. Free radical polymerization was initiated by $\mathrm{N}, \mathrm{N}, \mathrm{N}, \mathrm{N}-$ tetramethylethylenediamine (TEMED) and ammonium persulfate (APS) pair $(1.2 \mathrm{w} / \mathrm{w} \%$ TEMED and $1.2 \mathrm{w} / \mathrm{w} \%$ APS of the total weight of monomers and MBAA). The mixture was then cooled under ice bath for about 30 minutes. APS was added to the solution for the onset of reaction. The solution (4 ml) was aliquoted into glass columns, and frozen at $-12^{\circ} \mathrm{C}$ for 18 hours in a cryobath. The cryogels were allowed to thaw at room temperature while still in the glass columns before being washed with water; the gel matrix was kept in water and stored in the fridge till further use. Using the same monomers ratio to MBAA (6:1), five monomer concentrations - 6, 8,10,13 and $16 \mathrm{w} / \mathrm{v} \%$ were used to synthesize epoxy PHEMA cryogel samples. 


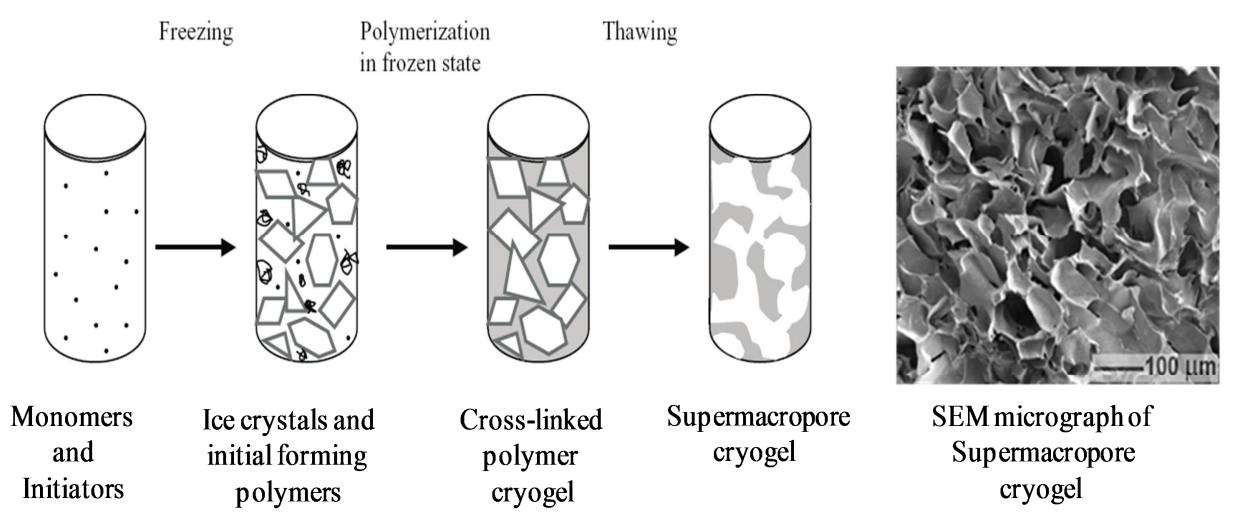

Figure 1. Schematic outline of the formation of cryogel and SEM micrograph of the cryogel.

\subsection{Scanning Electron Microscopy (SEM) Preparation}

The cryogel samples were fixed in $2.5 \%$ glutaraldehyde for 2 hours at room temperature. Then the samples were dehydrated in ethanol (0-50-75-99.5\%) and air dried overnight in the fume cupboard. The dried samples were coated with palladium and gold (40/60) and examined using a JOEL JSM-5600LV scanning electron microscope.

\subsection{Confocal Laser Scanning Microscopy (CLSM) Preparation}

The cryogel was taken out of the column and $\sim 1 \mathrm{~mm}$ sections were cut from the top, middle and bottom of the column. Each slice was stained with FITC (Fluorescein 5(6) - isothiocyanate) in $0.02 \mathrm{mg} / \mathrm{ml}$ in $0.1 \mathrm{M}$ sodium phosphate buffer, $\mathrm{pH} 7.0$ for 48 hours. Non-bound FITC was washed out with buffer and cryogel slices were observed under confocal laser scanning microscope (CLSM). The fluorescent images were obtained using a confocal laserscanning microscope, (Zeiss LSM410). A plantApochromat $20 \mathrm{x} / 0.5$ objective was used in all experiments. All images were taken at 512 X 512 pixels with pixel size of $0.999 \mu \mathrm{m}$ (for x-plane) and $0.98 \mu \mathrm{m}$ (for z-plane). The number of sections was in the range of 54-73. Images collected by CLSM were used for the calculation of porosity, pore sizes and wall thickness using the software image $\mathrm{J}$.

\subsection{Measurement of Mechanical Properties of Cryogel by Dynamic Mechanical Analyzer (DMA)}

\subsubsection{Measurement of Compressive Modulus of Cryogels}

The compression test on epoxy PHEMA cryogel was performed using the uniaxial compression test. The sample from top, middle and bottom section of the cryogel column, was sliced to approximately $10 \mathrm{~mm}$ diameter and $9 \mathrm{~mm}$ in height and placed on a compression plate as shown in Fig.2, then heated to $37^{\circ} \mathrm{C}$ and kept isothermal. An initial force of $0.01 \mathrm{~N}$ was applied to touch the sample surface and then increased gradually to $18 \mathrm{~N}$. The compressive modulus was determined as the slope of the stress-strain curve in the linear elastic region between $0-20 \%$ strain.

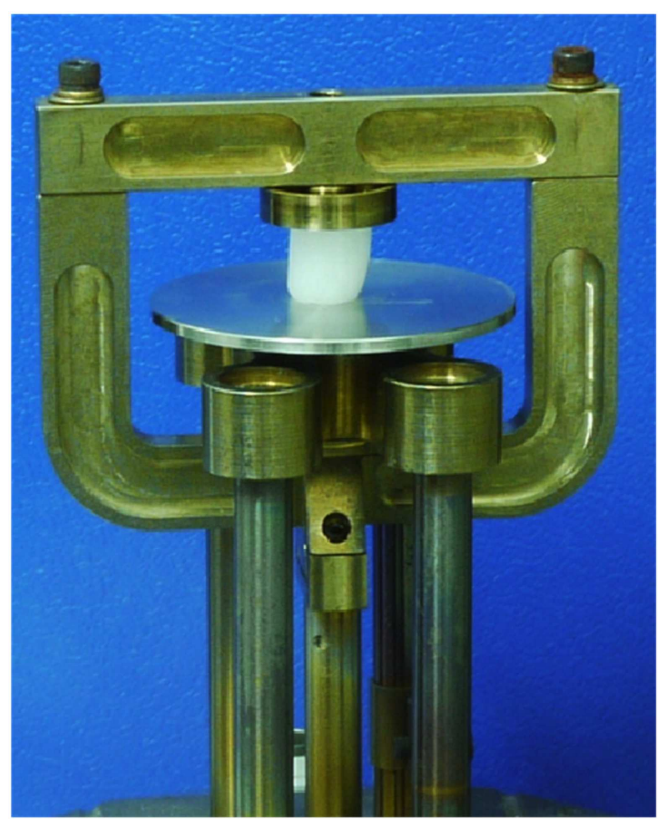

Figure 2. Slice of cryogel soaked with water on a compression plate.

\subsubsection{Creep Test of Cryogels}

A cryogel slice of approximately $10 \mathrm{~mm}$ diameter and 9 $\mathrm{mm}$ height was placed on the compression plate as shown in Fig.2. At constant temperature of $37^{\circ} \mathrm{C}$ and constant load (stress) of $0.2 \mathrm{~N}$ was applied. The sample was left for 10 minutes to recover after the release of constant load. The recovery strain versus time was measured.

\subsubsection{Measurement of the Strain Percentage Against Time with a Constant Stress When the Cryogel is Soaked with Water}

The sample was treated as mentioned in 2.4.2, a constant load of $0.2 \mathrm{~N}$ was applied on the cryogel slice for one minute and allowed to rehydrate for two minutes, and the cycle was repeated four times. 


\subsubsection{Yield Percentage and Swelling Degree of Cryogels}

Cryogel samples $0.5 \mathrm{ml}(\mathrm{n}=3)$ were put in an oven at $60^{\circ} \mathrm{C}$ overnight for drying. After drying until constant weight was reached, the mass of the dried sample was determined. The solid polymer fraction yield was calculated using equation 2 .

$$
\frac{\text { Mass of dry cryogel }}{\text { Initial mass of polymer (+crosslinker) }} X 100 \text { Equation } 2
$$

\subsubsection{Statistical Analysis}

All statistical analyses were performed by first using one-way analysis of variance (ANOVA, minitab version 15). Whenever ANOVA indicated the groups significantly different, a t-test for independent samples was performed. Samples were considered significantly different at $\mathrm{P} \leq 0.05$.

\section{Results and Discussions}

\subsection{Synthesis of Monolithic Cryogels}

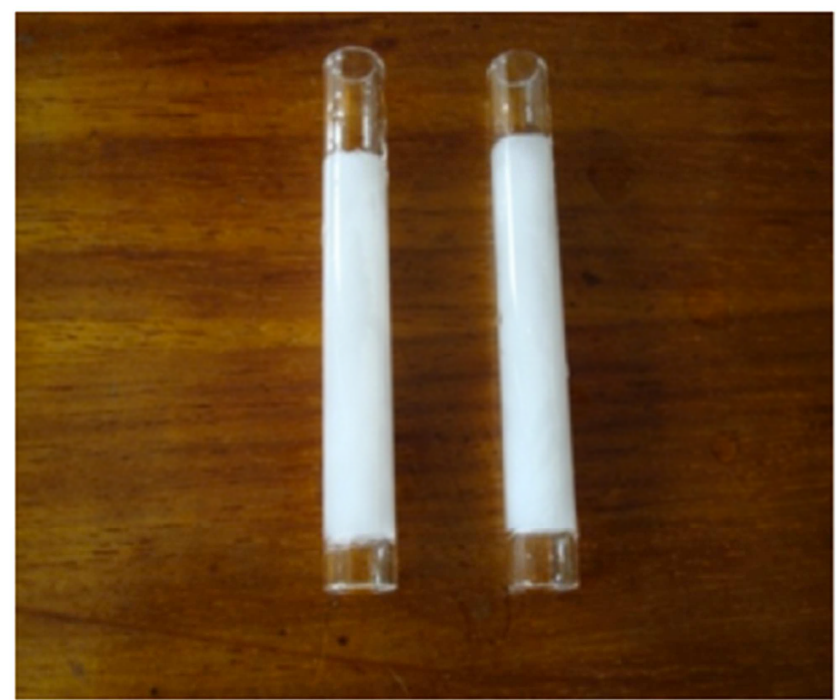

Figure 3. Cryogel in glass column after freezing, thawing and washing with water.

A range of continuous monolithic, cryogel columns was synthesized by co-polymerization of monomers in the frozen state. Fig 1 shows schematically the steps required for the formation of a cryogel. Epoxy-PHEMA monolithic cryogel synthesis success was very dependent on freezing gelation interaction. The most important step during the synthesis process was the rapid freezing of the reaction mixture; this takes place before the onset of gelation. Gels not different from traditional ones (hydrogel) are formed when gelation starts before freezing. In order to prevent the production of poor quality monolithic macroporous cryogels, freezing was facilitated. Both empty glass columns and the reaction systems (monomer mixture and initiator system) were ice cooled for at least 30 minutes before the reaction was initiated. The transfer of the reaction mixture into the empty glass columns was done rapidly to minimize gelation rate due to high temperatures. The cryogelation polymerization reaction began with the reaction between the initiator APS and the activator
TEMED to form free radicals. The free radical initiates the reaction of monomers HEMA, MBAAm and AGE (epoxy groups enhancer). MBAAm also acts as a cross-linker. Fig. 3 shows a monolithic cryogel after cryo polymerization in a glass column. The percentage recovery of monoliths per batch of 20 independent batches for $6 \%, 8 \%, 10 \%, 13 \%$ and $16 \%$ epoxy PHEMA was 97\%, 99\%, 98\%, 96\% and $97 \%$. This proves that the cryogelation process is highly reproducible.

\subsection{Physical Properties of Monolithic Cryogels Obtained After Cryopolymerization at $-12^{\circ} \mathrm{C}$}

Epoxy PHEMA cryogel produced were white, opaque, soft and spongy cylindrical blocks built up with systems of interconnected pore matrix. The monoliths appear relatively more opaque as monomer concentration increases in their wet state. It was possible to mechanically squeeze out water from the monoliths and the monoliths re-swell immediately when in contact with water. The spongy-like morphology, macropores interconnection and mechanical stability of epoxy PHEMA cryogel are typical characteristics of cryogels. However, the observed spongy and soft morphology was decreasing as monomer concentration increases. This probably could be attributed to the formation of thicker (denser) walls following increase of polymer content as monomer concentration in the reaction system was increased [2]. From observation, 16\% epoxy PHEMA cryogels appeared relatively rigid at their removal from the glass columns after thawing. On the other hand, 8\% epoxy PHEMA cryogels appeared less rigid and softer. In general, decreasing the initial monomer concentration below $6 \%$ would be associated with the production of fragile cryogels while increasing the initial monomer concentration above $16 \%$ could lead to the formation of rigid and less macroporous cryogels.

\subsection{Structure of Cryogel Obtained After Cryopolymerization at $-12^{\circ} \mathrm{C}$}

When cryogels are produced, polymerization occurs in the non-frozen fluids containing dissolved monomers and initiator. The ice crystals formed during freezing works as a pore agent. Thus, the shape and size of the ice crystals formed determine the shape and size of the pores formed after defrosting the sample. The freezing rate is determined by the starting temperature, which was $0^{\circ} \mathrm{C}$ in this situation, as all solutions before mixing were kept in an ice bath, and the freezing temperature was $-12^{\circ} \mathrm{C}$.

In this study, SEM and CLSM were used for pore structure analysis. Although SEM operates in dry states, the method is still appropriate for the macroporous epoxy PHEMA cryogels. As shown from the SEM micrographs (Fig: 4), the produced epoxy PHEMA monolithic cryogels were porous material of micrometre scale. Observation from SEM images (Fig: 4) shows that 8\% epoxy PHEMA monolithic cryogel has larger pores compared to $10 \%, 13 \%$ and $16 \%$. 

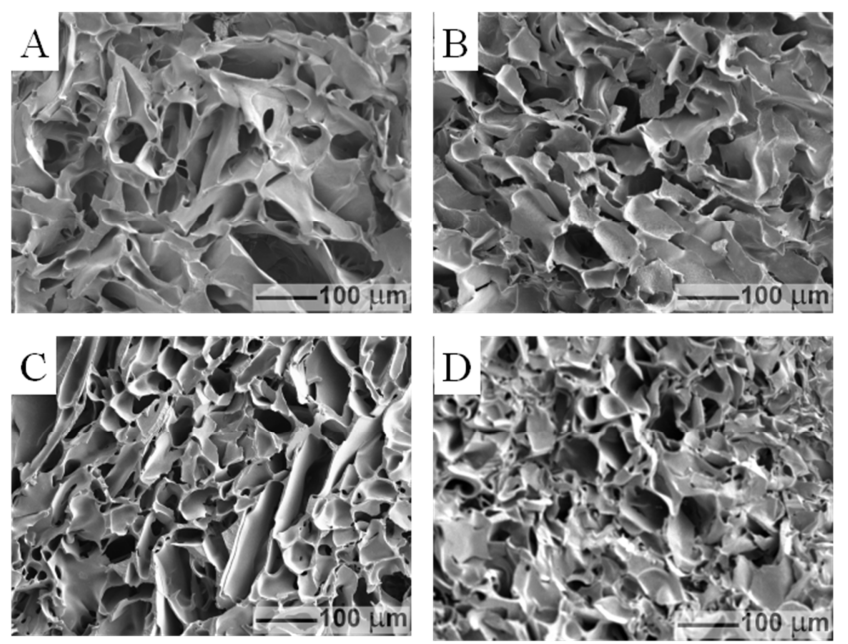

Figure 4. SEM microphotography of (A-D) 8\%,10\%,13\% and $16 \%$ of epoxy PHEMA cryogels.

Table 1. Polymerization yield of cryogels.

\begin{tabular}{ll}
\hline Sample & Yield percentage in water \\
\hline $8 \% \mathrm{w} / \mathrm{v}$ HEMA:AGE & $72 \pm 2.9$ \\
\hline
\end{tabular}

Table 1 presents the result of yield of polymerization of $8 \%$ w/v epoxy PHEMA cryogel. The cryogels were produced with high gelation yield of $\sim 72 \%$ for cryogel synthesized from co- polymerization of $8 \% \mathrm{w} / \mathrm{v}$ HEMA: AGE.

\subsection{Compressive Modulus of Cryogels}

As a result of the presence of large pores surrounded by dense polymer-pore walls, epoxy PHEMA cryogels are highly elastic and have spongy-like morphology. They can be easily compressed. The elastic and compression properties of the cryogel were determined by exerting physical stress on the gel, which was in turn used to calculate Young's modulus, which is a mathematical description of a substance's tendency to be deformed when force is applied to it. Fig.5 shows the compressive modulus of each cryogel sample of same size as calculated by analysing the stress and strain values of each cryogel by the DMA. The compressive modulus of epoxy PHEMA cryogel from 6, 8, 10 and $16 \%$ is approximately $1.8 \mathrm{kPa}, 3.4 \mathrm{kPa}, 5.1 \mathrm{kPa}$ and $8.5 \mathrm{kPa}$. These data could indicate that an increase in monomer concentration results in an increase in both polymer concentration in pore walls and the general strength of the macroporous gel backbone. Epoxy PHEMA cryogels are quite elastic materials with rather low value for the compressive modulus equal between 1.8-8.5 kPa. For comparison, the compressive modulus of soft tissues in human body is about 100-fold higher [28]. It is well demonstrated that the stress required for both 6 and $8 \%$ epoxy PHEMA cryogel to undergo compression is less than the stress required for 10 and $16 \%$. This infers that the 6 and $8 \%$ epoxy PHEMA cryogel is more elastic, spongier and soft. It was also observed that with the change in total monomer concentration from 6 to $16 \%$, the rigidity of cryogels was altered as shown in (Fig. 5). As the concentration increases, the sponginess and elasticity decrease, which in turn decreases compressibility and squeezability of the cryogel. The compressive modulus within the three different layers of cryogel for all samples from 6 to $16 \%$ showed they were almost similar within the layers. These results suggest that at all layers the gel has equivalent strength to withstand compression.

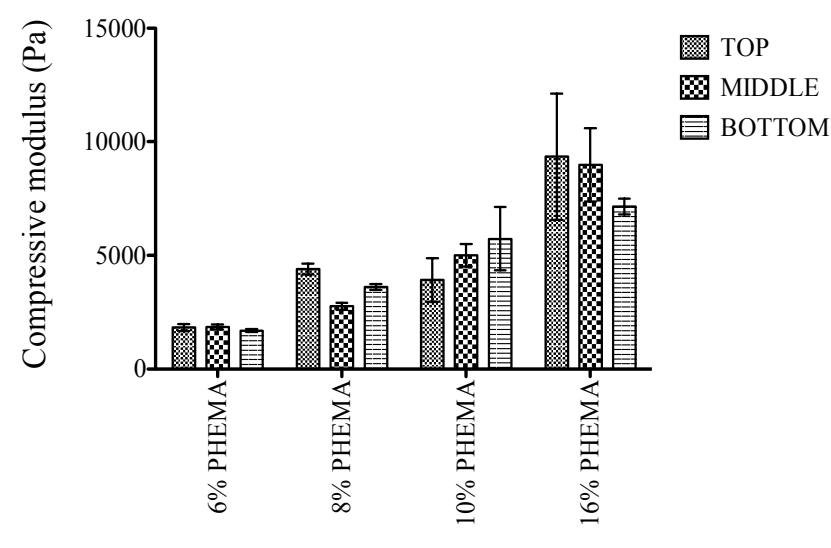

Monomer concentration

Figure 5. Comparative studies of mechanical strength of epoxy PHEMA. There is a significant difference in the compressive modulus when comparing $6 \%, 8 \%, 10 \%$ and $16 \%$ PHEMA cryogel with $P \leq 0.05$. (Mean \pm $S D n=6)$.

One of the potential applications of these cryogels that has been recently established is the detachment of bio particles, which are attached/adsorbed to the surface of cryogel [29]. This detachment of bio particles is facilitated by elastic deformation of cryogels. Thus, it can be said that elasticity of cryogel is an important factor for such applications.

\subsection{Creep-Recovery Analysis of Cryogels}

A creep recovery test involves the application of a finite 
stress to a sample whilst recording the strain response as a function of time. Following a defined period, the stress is removed and the strain recovery is recorded. In particular, the creep test provides information on the viscoelastic or elastic behaviour of materials. The strain recovery percentage of epoxy PHEMA cryogel 8, 10 and 16\% after compression to $0 \%$ for approximately one minute and released to recover for about 10 minutes is as shown in
(Fig.6) 100\%, (Fig.7) 97\% and (Fig.8) 96\%. The decrease in strain recovery as the monomer concentration increases demonstrates that increase in monomer concentration increases the cryogel stiffness, hence less elastic. The creep test shows cryogels have a spongy-like structure with most of the water located within large macropores. The mechanical removal of the water by squeezing the cryogel is due to the elasticity property of cryogel.

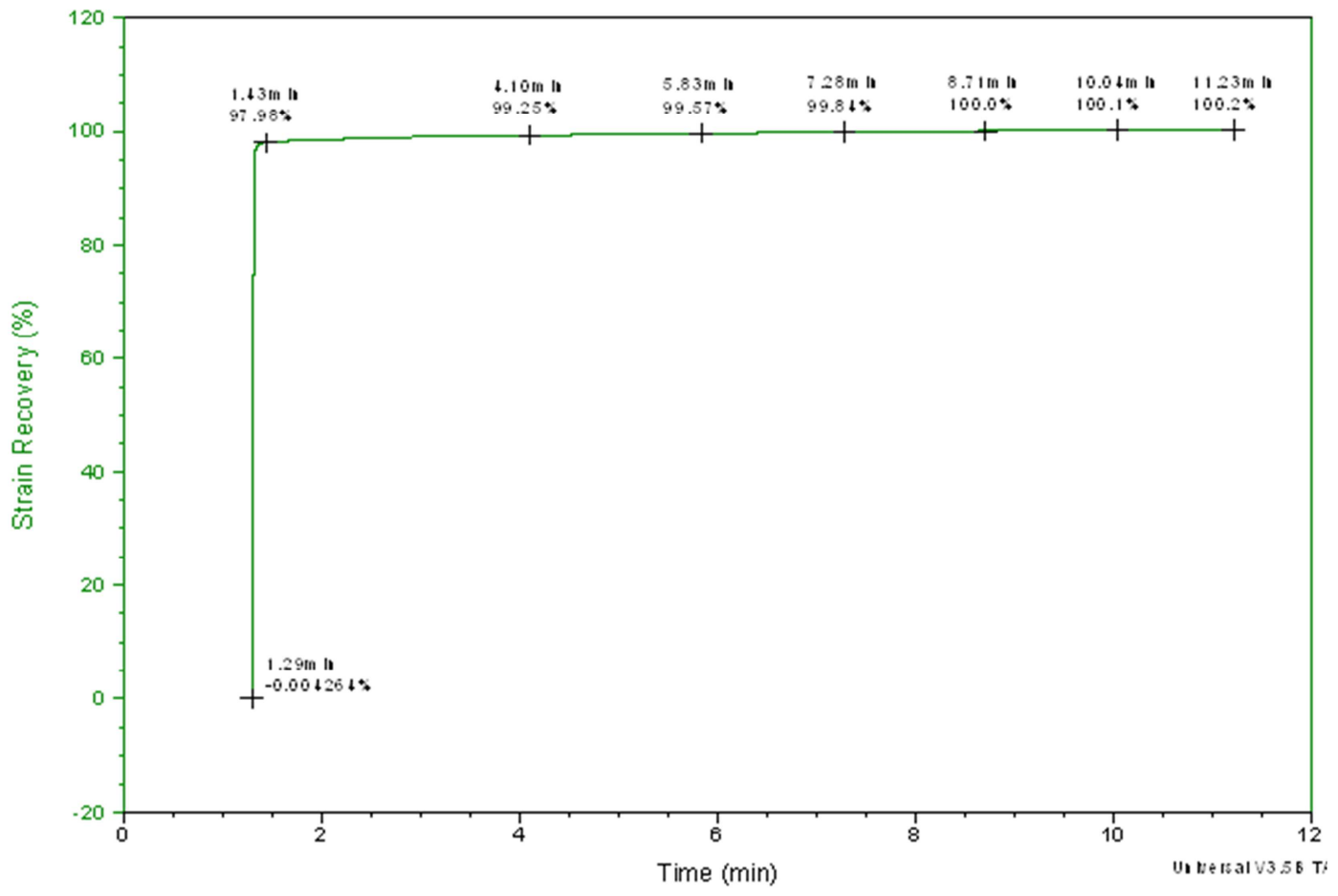

Figure 6. Creep test of 8\% epoxy PHEMA cryogel.

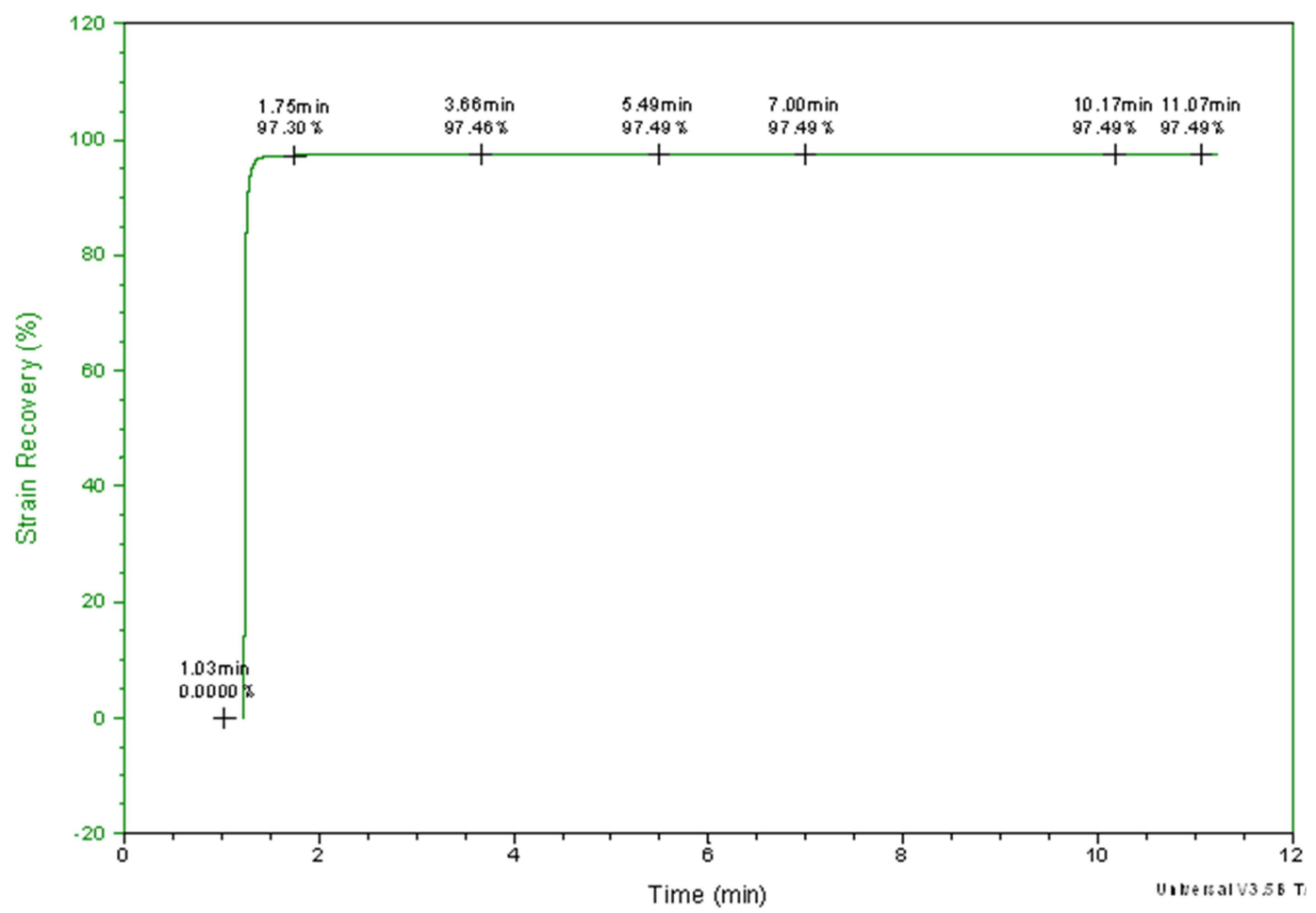

Figure 7. Creep test of $10 \%$ epoxy PHEMA cryogel. 


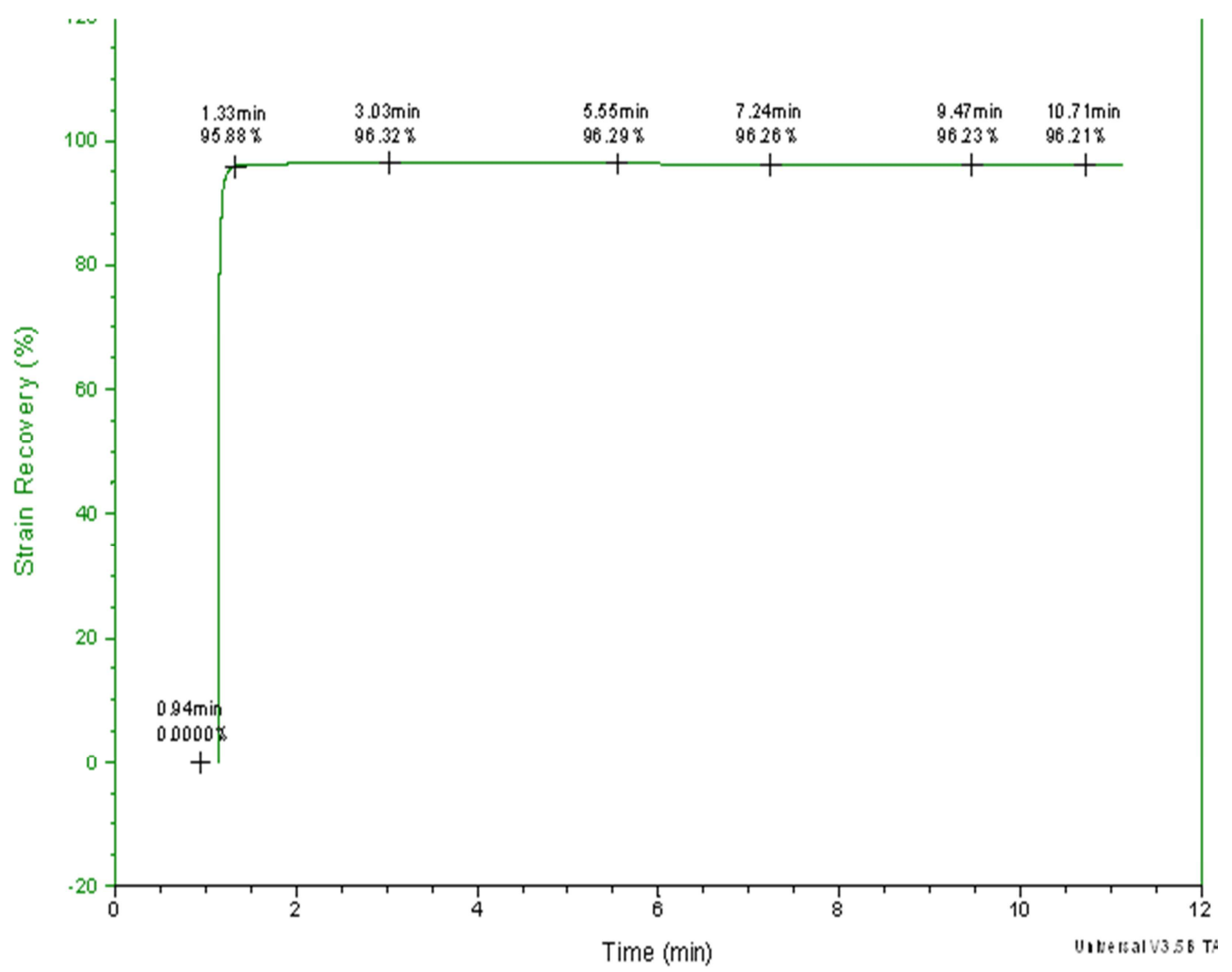

Figure 8. Creep test of 16\% epoxy PHEMA cryogel.

\subsection{Cyclic Compression/Stress-Relaxation Analysis of Cryogels}

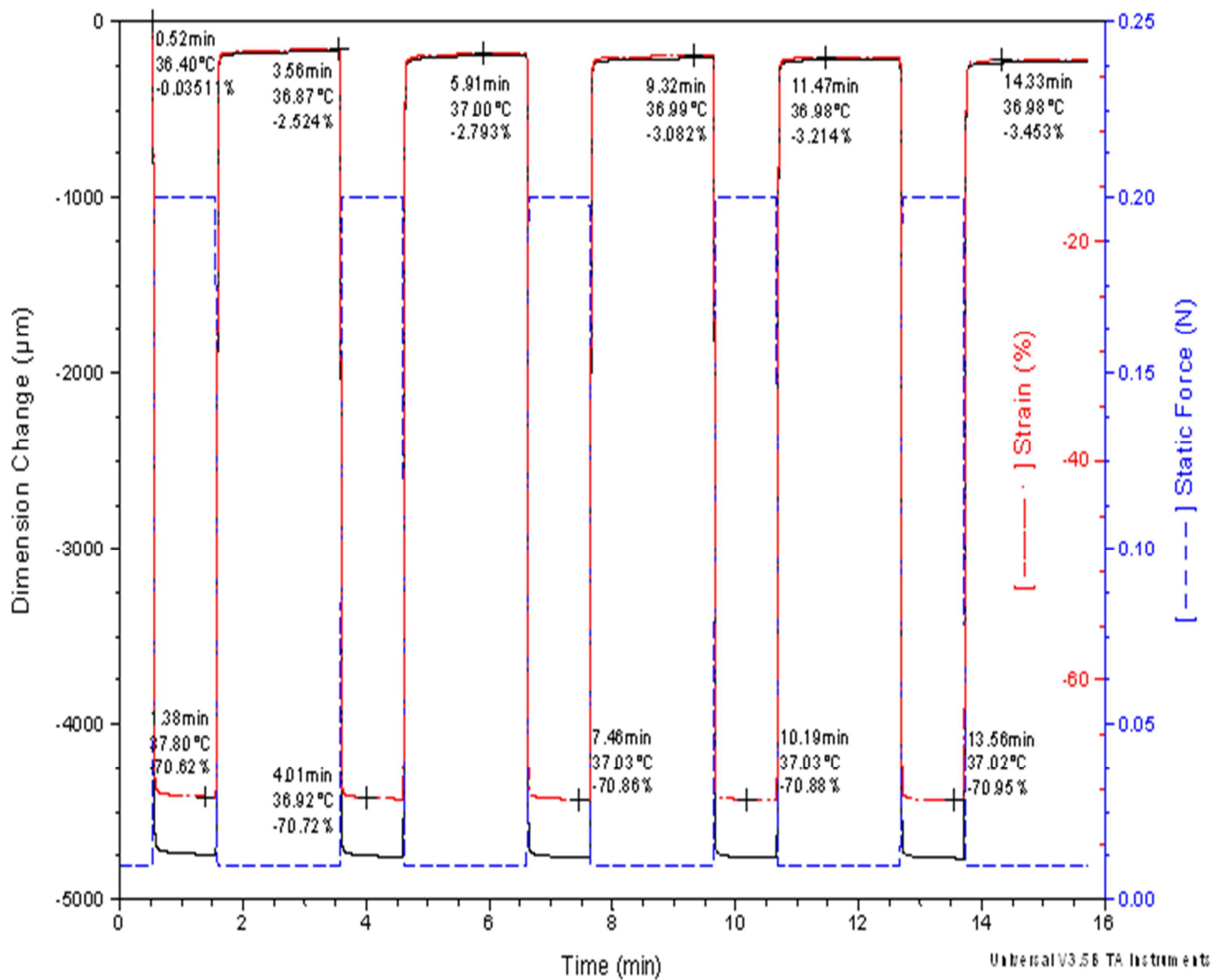

Figure 9. Cyclic compression of 6\% epoxy PHEMA cryogel. 


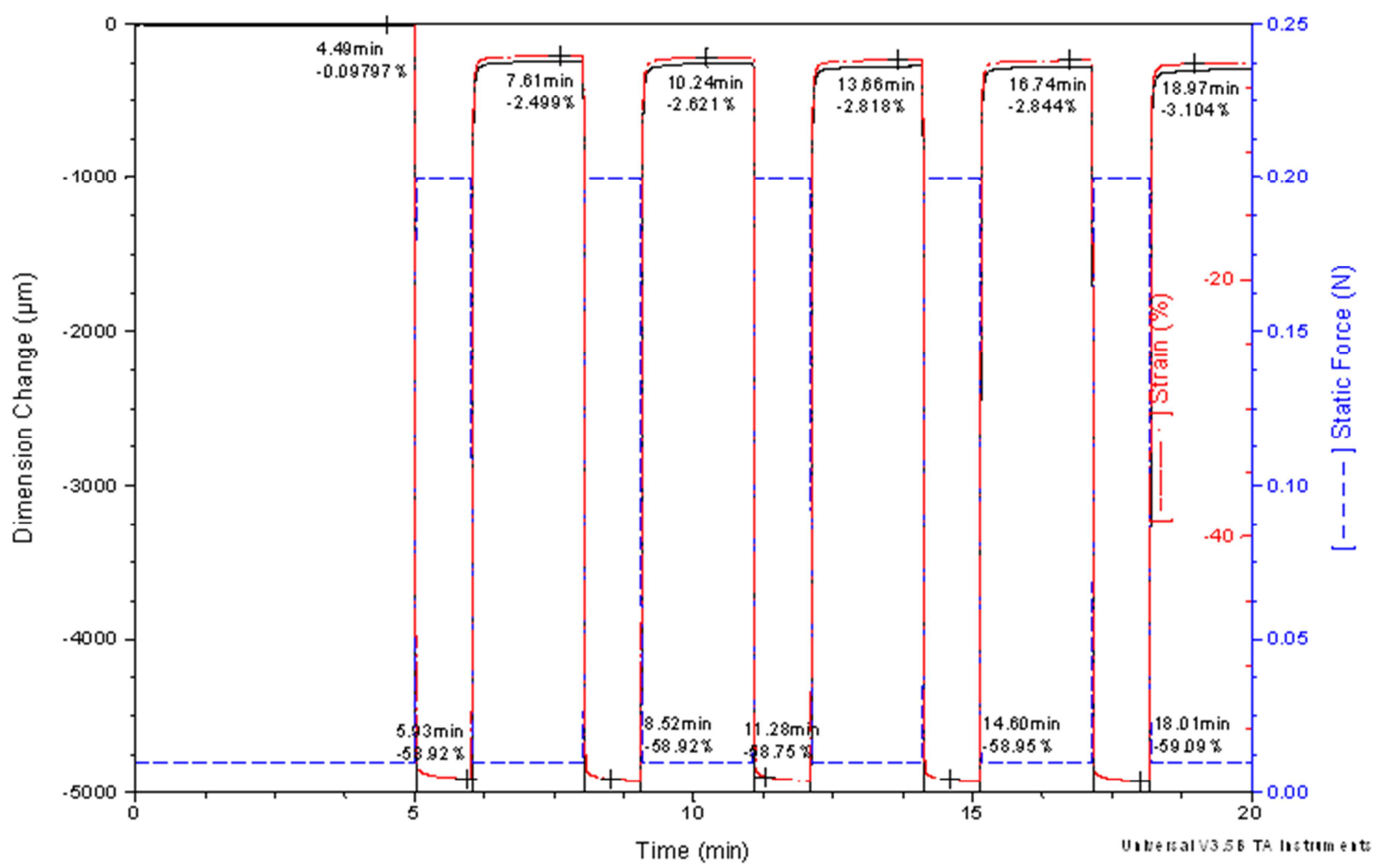

Figure 10. Cyclic compression of $8 \%$ epoxy PHEMA cryogel.

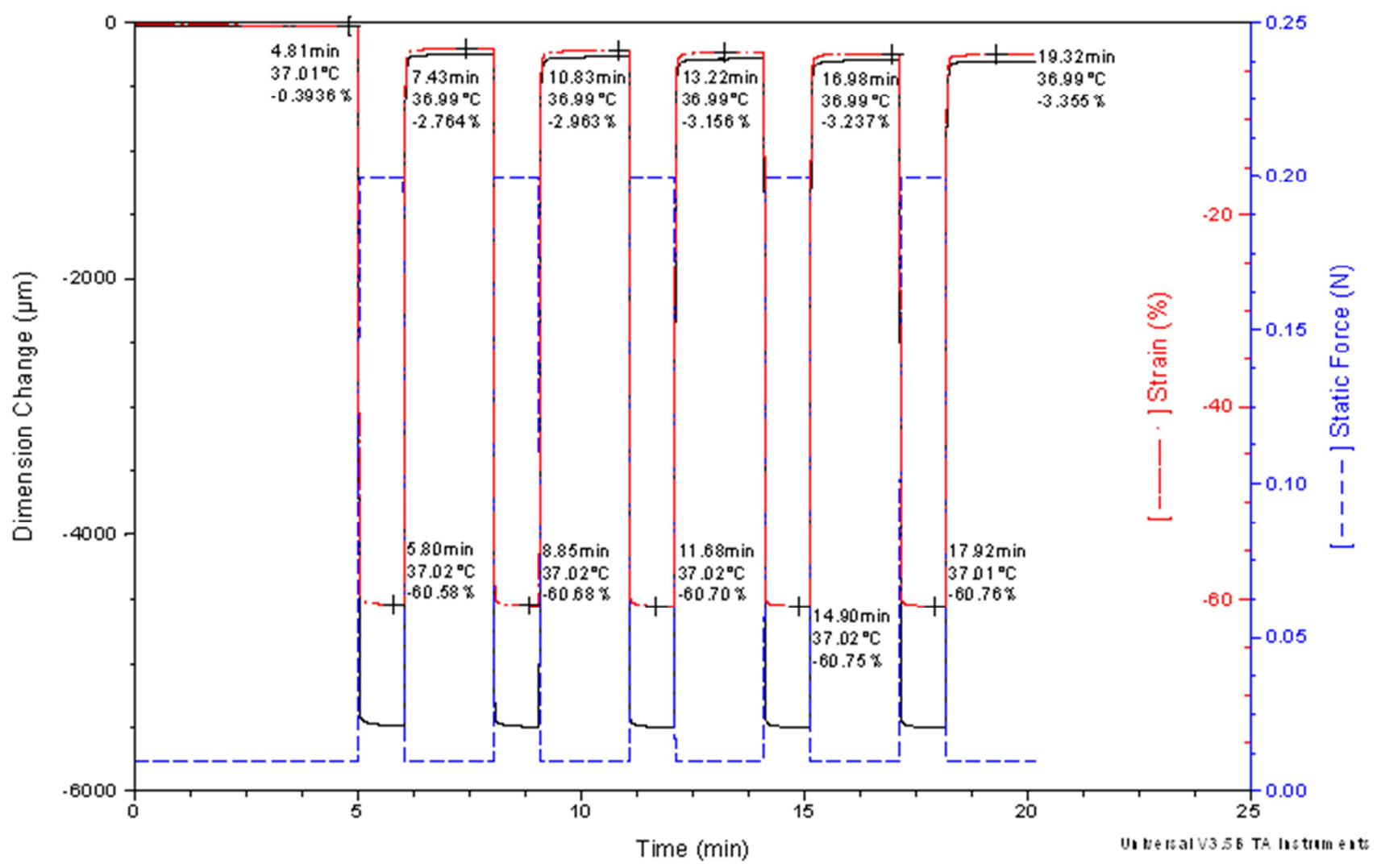

Figure 11. Cyclic compression of 10\% epoxy PHEMA cryogel. 


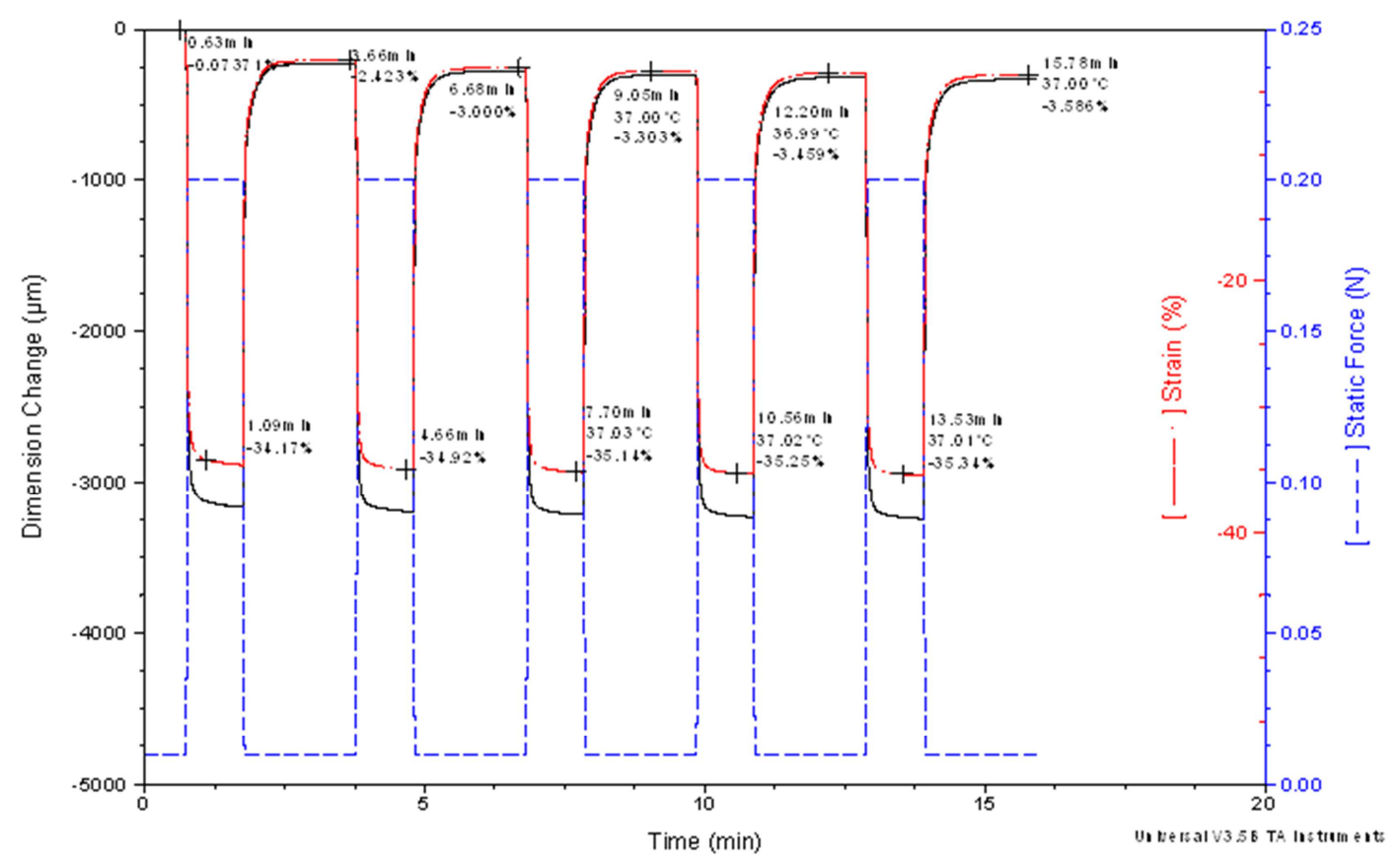

Figure 12. Cyclic compression of $16 \%$ epoxy PHEMA cryogel.

Creep-recovery and stress-relaxation methods are in principle the inverse of one another. During stress-relaxation testing, a sample is held at a constant deformation (strain) and the force required to maintain this strain is monitored as a function of time. Stress relaxation testing has been used extensively within the pharmaceutical industry. It has been used to probe the internal deformation of compressed materials and to gather information on the energy required for elastic and viscous deformation and hence to interpret the consolidation of different pharmaceutical compacts [30]. Macroporous cryogels are highly elastic and have a spongylike morphology, they can be easily compressed up to $70 \%$ of their original size without been destroyed mechanically. Epoxy PHEMA cryogels 6,8,10 and 16\% had approximately $97 \%$ recovery after applying a constant load of $0.2 \mathrm{~N}$ for one minute and released for two minutes and the cycle was repeated four different times. The results prove that cryogels did not lose their mechanical structure, as there was less than $1 \%$ difference in the recovery from the first cycle to the fifth cycle. However, the increase in monomer concentration affected the percentage of compressibility, this could be explained by the higher the monomer concentration the thicker the polymer walls, the smaller the interconnected pores and the more rigid and less elastic the cryogel material. Fig. 9 shows 6\% epoxy PHEMA cryogel was compressed to approximately 70\%, Fig. 10 shows 8\% epoxy PHEMA cryogel was compressed to approximately 59\%, Fig. 11 shows $10 \%$ epoxy PHEMA cryogel was compressed to approximately $60 \%$ and Fig. 12 shows $16 \%$ epoxy PHEMA cryogel was compressed to approximately $35 \%$ after applying a constant load of $0.2 \mathrm{~N}$ for one minute in a cyclic manner.

The results observed in this study suggest that the synthesized epoxy PHEMA cryogels are mechanically stable elastic and spongy materials, and monomer concentrations affect both physical and mechanical properties of cryogels.

\section{Conclusions}

The cryogelation technique has made it possible to produce monolithic materials with unique properties. The porosity of monoliths produced can be diverse in nature combined with flow through channels to structures with uniformly distributed interconnected macropores of $(\sim 10$ $\mu \mathrm{m}-120 \mu \mathrm{m}$ ) and twisted paths (main characteristics for freeze-thawed cryogels prepared via free radical polymerization). The cryogelation process was highly reproducible with over $90 \%$ recovery. Pore size and thickness of pore walls in porous epoxy PHEMA cryogels are regulated mainly by changing the monomer concentration in the reaction mixture. Macroporous cryogels produced from epoxy PHEMA using cryogelation have proved to be mechanically stable, elastic, and sponge-like materials that could undergo up to $70 \%$ compression and restore their properties ( $\sim 97 \%$ strain recovery). HEMA cryogels are soft materials and have potential as materials for cell separation and bio-separation in extracorporeal apheresis systems. These monoliths have an obvious potential as flowthrough filters for different applications in extracorporeal apheresis devices.

Acknowledgement: The authors' special thanks to $\mathrm{Dr}$ Aniela Leistner and Mr Andre Leistner of Polymerics $\mathrm{GmbH}$, Berlin, Germany for support with dynamic mechanical analyzer.

This work was financially supported by the European Commission Marie Curie Actions FP7 IAPP MONACO EXTRA project (218242) and University of Brighton PhD studentship. 


\section{References}

[1] Arvidsson, P., et al., Chromatography of microbial cells using continuous supermacroporous affinity and ion-exchange columns. Journal of Chromatography A, 2002. 977(1): p. 2738 .

[2] Plieva, F. M., et al., Pore structure in supermacroporous polyacrylamide based cryogels. Soft Matter, 2005. 1(4): p. 303-309.

[3] Dainiak, M. B., et al., Integrated isolation of antibody fragments from microbial cell culture fluids using supermacroporous cryogels. Journal of Chromatography A, 2004. 1045(1-2): p. 93-98.

[4] Arvidsson, P., et al., Direct chromatographic capture of enzyme from crude homogenate using immobilized metal affinity chromatography on a continuous supermacroporous adsorbent. Journal of Chromatography A, 2003. 986(2): p. 275-90.

[5] Plieva, F. M., H. Kirsebom, and B. Mattiasson, Preparation of macroporous cryostructurated gel monoliths, their characterization and main applications. Journal of Separation Science, 2011. 34(16-17): p. 2164-2172.

[6] Bowers, R. W. J. and B. J. Tighe, Studies of the ocular compatibility of hydrogels. A review of the clinical manifestations of spoliation. Biomaterials, 1987. 8(2): p. 83IN1.

[7] Rosiak, J., J. Olejniczak, and W. Pakala, Fast reaction of irradiated polymers. Crosslinking and degradation of polyvinylpyrrolidone. International Journal of Radiation Applications and Instrumentation. Part C. Radiation Physics and Chemistry, 1990. 36(6): p. 747-755.

[8] Dziubla, T. D., et al., Evaluation of porous networks of poly(2-hydroxyethyl methacrylate) as interfacial drug delivery devices. Biomaterials, 2001. 22(21): p. 2893-2899.

[9] Bajpai, A. K. and M. Shrivastava, Water sorption dynamics of a binary copolymeric hydrogel of 2-hydroxyethyl methacrylate (HEMA). Journal of Biomaterials Science, Polymer Edition, 2002. 13(3): p. 237-256.

[10] Lozinsky, V. I., et al., Polymeric cryogels as promising materials of biotechnological interest. Trends in Biotechnology, 2003. 21(10): p. 445-451.

[11] Savina, I. N., et al., Porous structure and water state in crosslinked polymer and protein cryo-hydrogels. Soft Matter, 2011. 7(9): p. 4276-4283.

[12] Ertürk, G. and B. Mattiasson, Cryogels-versatile tools in bioseparation. Journal of Chromatography A, 2014. 1357: p. 24-35.

[13] Gun'ko, V. M., I. N. Savina, and S. V. Mikhalovsky, Cryogels: morphological, structural and adsorption characterisation. Adv Colloid Interface Sci, 2013. 187-188: p. 1-46.

[14] Hanora, A., et al., Screening of peptide affinity tags using immobilised metal affinity chromatography in 96-well plate format. Journal of Chromatography A, 2005. 1087(1-2): p. $38-44$.
[15] Le Noir, M., et al., Macroporous molecularly imprinted polymer/cryogel composite systems for the removal of endocrine disrupting trace contaminants. Journal of Chromatography A, 2007. 1154(1-2): p. 158-164.

[16] Ingavle, G. C., et al., Affinity binding of antibodies to supermacroporous cryogel adsorbents with immobilized protein A for removal of anthrax toxin protective antigen. Biomaterials, 2015. 50: p. 140-153.

[17] Hajizadeh, S. and B. Mattiasson, Cryogels with Affinity Ligands as Tools in Protein Purification, in Affinity Chromatography, S. Reichelt, Editor. 2015, Springer New York. p. 183-200.

[18] Plieva , F., et al., Macroporous elastic polyacrylamide gels prepared at subzero temperatures: control of porous structure. Journal of Materials Chemistry, 2006. 16(41): p. 4065-4073.

[19] Jones, D. S., et al., Pharmaceutical applications of dynamic mechanical thermal analysis. Advanced Drug Delivery Reviews, 2012. 64(5): p. 440-448.

[20] Jones, D. S., A. D. Woolfson, and A. F. Brown, Textural, viscoelastic and mucoadhesive properties of pharmaceutical gels composed of cellulose polymers. International Journal of Pharmaceutics, 1997. 151(2): p. 223-233.

[21] Ferry, J. D., Viscoelastic Properties of Polymers. 1980: John Wiley and Sons.

[22] Ward , I. M. and D. W. Hardley An introduction to the mechanical properties of solid polymers. 1993: John Wiley and sons.

[23] Jones, D. S., Dynamic mechanical analysis of polymeric systems of pharmaceutical and biomedical significance. International Journal of Pharmaceutics, 1999. 179(2): p. 167178.

[24] Barnes, H. A., J. F. Hutton, and K. Walters, An introduction to rheology. 1996: Elsevier.

[25] Craig, D. Q. M. and F. A. Johnson, Pharmaceutical applications of dynamic mechanical thermal analysis. Thermochimica Acta, 1995. 248: p. 97-115.

[26] Patfoort, G., An introduction to physical, mechanical and rheological behaviour. 1974: Story -Scientia, Gent.

[27] Denizli, A., R. Say, and E. Piskin, Removal of aluminium by Alizarin Yellow-attached magnetic poly(2-hydroxyethyl methacrylate) beads. Reactive and Functional Polymers, 2003. 55(1): p. 99-107.

[28] Hollister, S. J., Porous scaffold design for tissue engineering. Nature Materials, 2005. 4(7): p. 518-524.

[29] Dainiak, M. B., et al., Detachment of affinity-captured bioparticles by elastic deformation of a macroporous hydrogel. Proceedings of the National Academy of Sciences, 2006. 103(4): p. 849-854.

[30] Casahoursat, L., G. Lemagnen, and D. Larrouture, The Use of Stress Relaxation Trials to Characterize Tablet Capping. Drug Development and Industrial Pharmacy, 1988. 14(15-17): p. 2179-2199. 